\title{
Hábitos alcoólicos e protecção cardiovascular no Centro de Saúde de Barão do Corvo
}

Martinho Almeida, ${ }^{*}$ Vânia Cardoso, ${ }^{*}$ Pedro Araújo, ${ }^{*}$ Cristiana Leite, ${ }^{*}$ Patrícia Marques, ${ }^{*}$ Andrea Lobão, ${ }^{*}$ Filomena Vidal,** Maria Elvira Pinto,***

\section{RESUMO}

Objectivos: Caracterizar hábitos de consumo de bebidas alcoólicas dos utentes utilizadores do Centro de Saúde Barão do Corvo (CSBC) e verificar se existe associação entre hábitos alcoólicos moderados e o risco global cardiovascular (RGC), calculado pelas tabelas SCORE.

Tipo de estudo: Estudo observacional, transversal e analítico.

Local: Ambulatório de medicina geral e familiar.

População do estudo: utentes utilizadores do CSBC com idade $\geq$ a 18 anos.

Métodos: Colheita de dados através de entrevista pessoal, em Maio e Junho de 2007, no CSBC, Vila Nova de Gaia. Amostra de conveniência $(n=502)$. Usado o teste $\chi^{2}$. Nível de significância: 0,05 .

Resultados: $51,8 \%$ dos inquiridos consumiram álcool alguma vez na vida, e $46 \%$ no último ano. O consumo é maior entre o sexo masculino, indivíduos mais idosos, reformados, viúvos e com escolaridade inferior ao $3^{\circ}$ ciclo. $63,7 \%$ dos inquiridos iniciaram o consumo antes dos 20 anos. 39,6\% eram consumidores moderados, e 6,8\% consumidores pesados. A proporção de inquiridos com RGC superior a $5 \%$ aumenta com o consumo de álcool.

Conclusão: Comparando com a população portuguesa, verifica-se menor consumo de álcool alguma vez na vida (51,8\% vs $76 \%$ ) e no último ano (46\% vs 59\%). Como na população portuguesa, a idade do primeiro contacto situa-se maioritariamente entre os 15 e os 19 anos. A duração média do consumo é superior à da população portuguesa. Os resultados apoiam a hipótese da curva dose-resposta para o consumo de bebidas alcoólicos ser em J e não em U. Assim, parece mais prudente não aconselhar o consumo de álcool pelos seus benefícios cardiovasculares e recomendar a diminuição deste consumo aos consumidores habituais.

Palavras-chave: Hábitos Alcoólicos; Protecção Cardiovascular.

\section{INTRODUÇÃO}

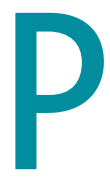

ortugal apresenta dos maiores consumos de bebidas alcoólicas e prevalência de problemas ligados ao álcool (PLA), entre os países membros da União Europeia. Calcula-se que cerca de $60 \%$ da população adulta portuguesa $(80 \%$ dos homens e $50 \%$ das mulheres) corresponda ao grupo dos bebedores regulares de álcool, sendo neste grupo que se encontram os bebedores excessivos, muitas vezes não identificados. Estima-se que cerca de $10 \%$ da população nacional apresente graves incapacidades liga-

\footnotetext{
*Internos de Medicina Geral e Familiar

**Assistente de Medicina Geral e Familiar

***Assistente Graduada de Medicina Geral e Familiar

Centro de Saúde de Barão do Corvo (CSBC), Vila Nova de Gaia
}

das ao álcool. ${ }^{1}$

O consumo de álcool está relacionado com as principais causas de morte em Portugal, nomeadamente doenças cardiovasculares (principalmente AVCs) e oncológicas (boca, faringe, laringe e esófago), acidentes rodoviários, suicídios (nos homens), cirrose hepática e doença pancreática. ${ }^{1}$

O efeito protector cardiovascular do consumo crónico de álcool em quantidades ligeiras a moderadas (cerca de $15 \mathrm{~g}$ por dia nas mulheres e entre $15 \mathrm{a} 30 \mathrm{~g}$ por dia nos homens) é reconhecido desde o fim da década de 80, estando associado a uma menor incidência de doença coronária e mortalidade cardiovascular., ${ }^{2,3}$

Alguns estudos afirmam ainda que os consumidores de baixo risco apresentam um risco de mortalidade car- 
diovascular inferior quando comparados aos abstémios e consumidores de risco de bebidas alcoólicas. ${ }^{4}$

Este fenómeno foi inicialmente denominado como o «Paradoxo Francês», em alusão a estudos epidemiológicos que revelaram que os habitantes de certas regiões francesas, com consumo de baixo risco de vinho, tinham menor incidência de doença coronária quando comparados com habitantes de outros países com hábitos dietéticos semelhantes, mas que não consumiam bebidas alcoólicas nessa proporção. ${ }^{5}$

Apesar de os mecanismos responsáveis pela protecção cardiovascular do consumo de baixo risco de álcool não terem ainda sido totalmente clarificados, dados recentes apontam para que, nesta protecção, ocorre a influência e conjugação de várias acções a nível bioquímico e molecular: modificações favoráveis no metabolismo lipídico (aumento das HDL em cerca de 30\%, aumento da Apolipoproteina A1 e A2, diminuição das LDL oxidadas), diminuição da agregação plaquetar, diminuição da homocisteína, aumento da fibrinólise, melhoria da função endotelial e vasodilatação arterial mediada pela libertação de NO, entre outros.

O Centro de Saúde Barão do Corvo (CSBC) abrange a freguesia de Santa Marinha, em Vila Nova de Gaia, onde se situam as caves do Vinho do Porto, sendo muito comuns os PLA, individuais, na família, no trabalho e na comunidade.

Assim, este estudo pretende caracterizar os hábitos de consumo de bebidas alcoólicas na população de utilizadores do CSBC, e ainda, apesar de o efeito protector cardiovascular do consumo de baixo risco de álcool estar bem estabelecido, verificar se existe associação entre os hábitos alcoólicos e o Risco Global Cardiovascular (RGC), calculado pelas tabelas derivadas do projecto SCORE (Systematic Coronary Risk Evaluation). ${ }^{6}$

\section{MÉTODOS}

Foi realizado um estudo, observacional, transversal e analítico, no âmbito do Projecto de Prevenção Cardiovascular «Sorri Coração», com colheita de dados em Maio e Junho de 2007, no CSBC, Vila Nova de Gaia, Portugal.

A população do estudo foi composta pelos utentes utilizadores $^{7}$ do CSBC, com idade igual ou superior a 18 anos, tendo sido excluídas mulheres grávidas ou com história de parto há menos de um ano.
Foi utilizada uma amostra não-aleatória de conveniência. Os utentes presentes na sala de espera das consultas dos diversos pisos do Centro de Saúde foram convidados a participar no estudo e incluídos no caso de aceitarem. A dimensão da amostra foi calculada, para uma prevalência esperada de $5 \%$ de diabetes mellitus, com nível de precisão de $2 \%$ e intervalo de confiança de $95 \%$. Para uma população de aproximadamente 60.000 utentes foi calculada uma amostra de 502 indivíduos.

A colheita de dados foi efectuada através de uma entrevista pessoal, com preenchimento de um questionário adaptado do «Canadian Heart Health Survey". ${ }^{8}$ Foi garantido o anonimato e a confidencialidade dos dados.

As variáveis estudadas foram: sexo, idade, escolaridade, estado civil, situação profissional, consumo de bebidas alcoólicas alguma vez na vida, consumo de bebidas alcoólicas no último ano, idade de início do consumo de álcool, número de unidades de bebidas alcoólicas consumidas por dia, hábitos tabágicos actuais, pressão arterial sistólica, colesterolemia capilar, antecedentes pessoais de doença coronária, doença cerebrovascular e diabetes mellitus.

O RGC foi calculado pelas tabelas do projecto SCO$\mathrm{RE}$, a partir das variáveis sexo, idade, hábitos tabágicos actuais, pressão arterial sistólica e colesterolemia capilar. Como recomendado pela Direcção-Geral da Saúde (DGS): foram usadas as tabelas de baixo risco; os utentes com antecedentes de doença cardíaca isquémica foram excluídos; para os utentes diabéticos, o risco foi multiplicado por 2 nos homens e por 4 nas mulheres; no caso de antecedentes de AVC/AIT, o risco foi multiplicado por 1,5.9.10

Os hábitos de consumo de bebidas alcoólicas foram auto-declarados pelo utente, durante a entrevista pessoal, assim como os hábitos tabágicos e os antecedentes pessoais de doença coronária, doença cerebrovascular e diabetes mellitus.

Foram considerados abstémios os indivíduos que não consumiram bebidas alcoólicas no último ano. Foi definido como consumo de baixo risco de bebidas alcoólicas, 2 ou menos doses diárias para as mulheres, 3 ou menos doses diárias para os homens (uma dose de álcool correspondendo a cerca de 8 a 13 g). Foram considerados consumidores de risco e nocivos, aqueles 
com consumos superiores aos definidos como de baixo risco.

A medição da pressão arterial foi efectuada duas vezes, após 5 minutos de repouso, na posição sentada, e, 20 minutos depois, no final da aplicação do questionário, utilizando um esfigmomanómetro OMRON M4-1.

O colesterol total foi medido com sangue capilar fresco, utilizando o sistema Accutrend ${ }^{\circledR}$ Cholesterol.

Os dados foram tratados utilizando o SPSS 15.0 e o Excel. Foi utilizado o teste de Wald-Wolfowitz, para sequências aleatórias, o teste de ajustamento do $\chi^{2}$, para comparação da amostra com a população em estudo, e o teste de independência do $\chi^{2}$, para a comparação de proporções. ${ }^{11,12}$

Foi adoptado um nível de significância de 0,05.

\section{RESULTADOS}

Dos 502 utentes entrevistados, 33\% eram do sexo masculino e $67 \%$ do sexo feminino; a idade dos inquiridos variou entre os 18 e os 91 anos, com uma idade média de 55,6 anos e uma mediana de 57 anos de idade; metade dos inquiridos tinham idades compreendidas entre os 44 e os 69 anos.

Quando comparada a amostra com a população de utilizadores do CSBC, não foram encontradas diferenças estatisticamente significativas em relação às variáveis sexo e grupo etário (Quadro I).

Dos utentes inquiridos, $51,8 \%$ já tinham consumido álcool alguma vez na vida, e $46 \%$ tiveram consumos no último ano. A ocorrência de consumo de álcool é supe-

\begin{tabular}{|c|c|c|c|c|}
\hline \multicolumn{5}{|c|}{$\begin{array}{l}\text { QUADRO I. Distribuição por sexo e grupo etário } \\
\text { dos utentes da amostra e da população de } \\
\text { utilizadores do CSBC }\end{array}$} \\
\hline & \multicolumn{2}{|c|}{ Amostra } & \multirow[b]{2}{*}{ População (\%) } & \multirow[b]{2}{*}{$\mathrm{p}^{*}$} \\
\hline & $\%$ & $\mathrm{n}$ & & \\
\hline \multicolumn{5}{|l|}{ Sexo } \\
\hline Homens & 33 & 165 & 35,4 & \multirow{2}{*}{0,236} \\
\hline Mulheres & 67 & 337 & 64,6 & \\
\hline \multicolumn{5}{|l|}{ Grupo etário } \\
\hline 19 a 44 & 25,7 & 129 & 27,4 & \multirow{3}{*}{0,631} \\
\hline 45 a 64 & 40,8 & 204 & 39,9 & \\
\hline$\geq 65$ & 33,8 & 169 & 34,7 & \\
\hline
\end{tabular}

*teste de ajustamento do $\chi^{2}$ rior nos homens quando comparada à das mulheres, tanto no último ano (69\% versus $35 \%$ ), como alguma vez na vida ( $78 \%$ versus $39 \%)$. Verifica-se também que, com o aumento da idade aumenta o número de indivíduos que consumiram álcool alguma vez na vida (Quadro II).

As características sociais dos indivíduos com história de consumo de bebidas alcoólicas alguma vez na vida encontram-se descritas no Quadro III. Verifica-se que o consumo de bebidas alcoólicas é mais frequente entre os indivíduos reformados, viúvos e com uma escolaridade inferior ao $3^{\circ}$ ciclo.

Considerando os utentes com história de consumo de álcool, $63,7 \%$ tiveram o primeiro consumo antes dos 20 anos; $52,7 \%$ dos homens iniciaram o consumo de bebidas alcoólicas entre os 15 e os 19 anos, e $66,4 \%$ das mulheres entre os 15 e os 24 anos (Figura 1).

A idade média do primeiro consumo foi de 16 anos para os homens e de 20 anos para as mulheres, sendo a média da duração do consumo de 41 anos (Quadro IV).

Quando distribuídos, os indivíduos da amostra, segundo o tipo de consumo - abstémios, consumidores de baixo risco e consumidores de risco e nocivos -, observa-se que $39,6 \%$ são consumidores de baixo risco e $6,8 \%$ consumidores de risco e nocivos (Figura 2). Verifica-se ainda que, entre os homens, $52,1 \%$ são consumidores de baixo risco e $17,6 \%$ consumidores de risco e nocivos e as mulheres são em maior percentagem abstémias (65\%).

\begin{tabular}{|c|c|c|c|c|}
\hline \multicolumn{5}{|c|}{$\begin{array}{l}\text { QUADRO II. Distribuição dos utentes da amostra com } \\
\text { história de consumo de álcool alguma vez na vida e no } \\
\text { último ano, por sexo e grupo etário }\end{array}$} \\
\hline & \multicolumn{2}{|c|}{ Uma vez } & \multicolumn{2}{|c|}{ Último ano } \\
\hline & $\%$ & $\mathbf{n}$ & $\%$ & $\mathbf{n}$ \\
\hline Sexo & & & & \\
\hline Masculino & 78 & 129 & 69 & 114 \\
\hline Feminino & 39 & 131 & 35 & 117 \\
\hline Grupo etário & & & & \\
\hline 18 a 34 & 19 & 12 & 17 & 11 \\
\hline 35 a 64 & 55 & 149 & 50 & 135 \\
\hline$\geq 65$ & 59 & 99 & 50 & 85 \\
\hline Total & 51,8 & 260 & 46 & 231 \\
\hline
\end{tabular}




\begin{tabular}{l|c|c}
\hline \multicolumn{2}{|l}{ QUADRO III. Distribuição dos utentes com história de } \\
consumo de álcool, alguma vez na vida, segundo a \\
situação profissional, estado civil e escolaridade \\
\multicolumn{2}{|c|}{ n } & $\%$ \\
\hline \multicolumn{2}{|l|}{ Situação profissional } & \\
Empregado & 92 & 46,2 \\
Desempregado & 30 & 52,6 \\
Doméstica & 20 & 51,3 \\
Estudante & 2 & 22,2 \\
Reformado & 116 & 58,9 \\
\hline Estado civil & & \\
Solteiro & 33 & 49,3 \\
Divorciado/Separado & 14 & 39 \\
Casado/União de Facto & 181 & 52,8 \\
Viúvo & 32 & 57,1 \\
\hline Escolaridade & & \\
Iletrado & 12 & 54,4 \\
$1^{\circ}$ ciclo & 140 & 54,3 \\
$2^{\circ}$ ciclo & 34 & 61,8 \\
$3^{\circ}$ ciclo & 29 & 46 \\
Secundário & 29 & 40,3 \\
Superior & 15 & 48,4 \\
\hline
\end{tabular}

Distribuindo os indivíduos da amostra, considerando o tipo de consumo, pelo RGC calculado pelas tabelas SCORE, verifica-se que entre os abstémios existe uma maior proporção de indivíduos com baixo risco cardiovascular (risco inferior a 5\%); nos consumidores de baixo risco a distribuição assume uma forma mais plana, existindo uma maior proporção de indivíduos com alto risco cardiovascular (risco superior ou igual a $5 \%$ ); entre os consumidores de risco, a proporção de indivíduos com alto risco cardiovascular é ainda maior (Figura 3).

A proporção de inquiridos com risco cardiovascular superior ou igual a 5\% aumenta com o consumo de bebidas alcoólicas, sendo as diferenças estatisticamente significativas (Quadro V).

Foi testada a associação entre o consumo de bebidas alcoólicas e os factores de risco cardiovascular modificáveis incluídos no cálculo do SCORE. Verificou-se existir associação estatisticamente significativa com os hábitos tabágicos e com a pressão arterial. Não se encontrou associação com a colesterolemia capilar, observando-se, no entanto, valores de colesterolemia

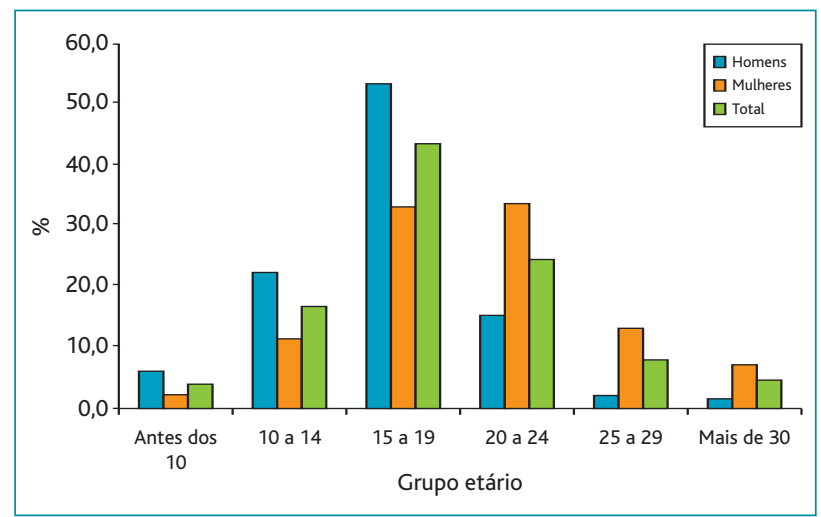

Figura 1. Distribuição dos utentes com história de consumo de álcool segundo a idade do $1^{\circ}$ consumo.

\begin{tabular}{|c|c|c|c|}
\hline & \multicolumn{2}{|c|}{$1^{\circ}$ consumo } & \multirow[b]{2}{*}{ Duração } \\
\hline & Homens & Mulheres & \\
\hline Média & 16,2 & 20,1 & 41 \\
\hline Desvio padrão & 4,3 & 7,6 & 15,8 \\
\hline Máximo & 32 & 68 & 79 \\
\hline Mínimo & 5 & 8 & 2 \\
\hline
\end{tabular}

inferiores entre os consumidores de baixo risco de bebidas alcoólicas (Quadro VI).

\section{DISCUSSÃO}

Pelo facto de a selecção dos participantes ter sido não-aleatória de conveniência, a amostra obtida poderia não ser representativa da população em estudo, limitando assim a extrapolação de resultados. No sentido de minimizar esta limitação, houve o cuidado de verificar se eram satisfeitas as condições que permitem aplicar os métodos estatísticos utilizados. Foi aplicado o teste de sequência de Wald-Wolfowitz ao sexo dos inquiridos $(\mathrm{p}<0,05)$, permitindo afirmar que, apesar de a colheita dos dados ter sido não-aleatória, a sequência de dados obtida é aleatória. De seguida, foi aplicado o teste de ajustamento do $\chi^{2}$ aos parâmetros sexo e idade da amostra e da população de utilizadores do CSBC, disponibilizados a partir do Sistema de Informação nas Unidades de Saúde (SINUS). Não foram encontradas diferenças estatisticamente significativas relativamente 


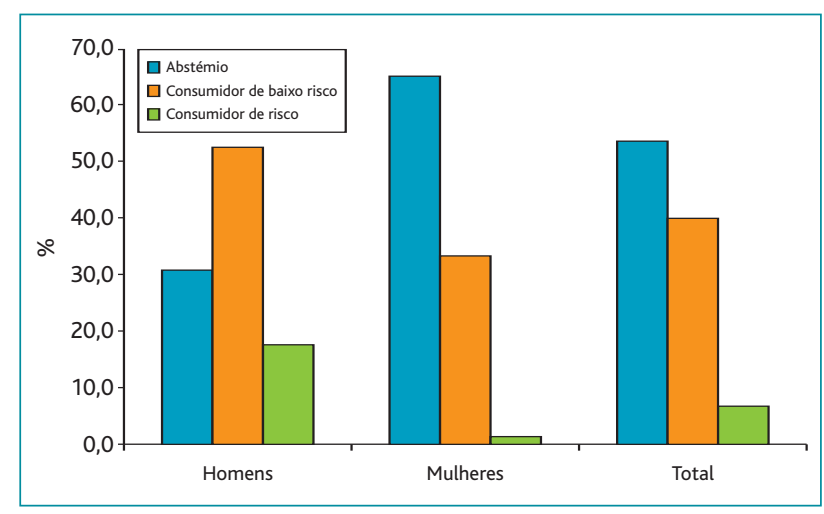

Figura 2. Distribuição dos inquiridos segundo o tipo de consumo

aos parâmetros sexo e idade ( $\mathrm{p}=0,236$ e $\mathrm{p}=0,631$, respectivamente), entre a amostra e a população dos utilizadores do CSBC, permitindo assim a extrapolação dos resultados.

Outras limitações do estudo foram a tradução e adaptação do questionário utilizado, não validado para a língua portuguesa, pelo que pode ter existido um viés de medição.

A colheita dos dados foi precedida por um estudo piloto, os entrevistadores foram treinados e foram utilizados instrumentos de medição calibrados e automáticos, tentando-se assim diminuir possíveis viéses do entrevistador e de medição.

Para a avaliação do RGC, foi aplicado o teste SCORE, por este reflectir no seu resultado algumas das modificações moleculares e bioquímicas pelas quais ocorre a cardioprotecção conferida pelo consumo de baixo risco de bebidas alcoólicas (aumento das HDL em cerca de $30 \%$, aumento da Apolipoproteina A1 e A2, diminuição das LDL oxidadas, melhoria da função endotelial e vasodilatação arterial mediada por NO, entre outros), através de alterações do padrão lipídico e da pressão ar-

\begin{tabular}{|c|c|c|c|}
\hline \multicolumn{4}{|c|}{$\begin{array}{l}\text { QUADRO V. Distribuição dos utentes em baixo e alto } \\
\text { risco cardiovascular, segundo o tipo de consumo }\end{array}$} \\
\hline & $<5 \%$ & $5 \%$ ou + & \multirow{4}{*}{$p<0,01^{*}$} \\
\hline Abstémio & $84,2 \%$ & $15,8 \%$ & \\
\hline Baixo risco & $72,1 \%$ & $27,9 \%$ & \\
\hline Nocivo & $50,0 \%$ & $50,0 \%$ & \\
\hline
\end{tabular}

*teste do $\chi^{2}$

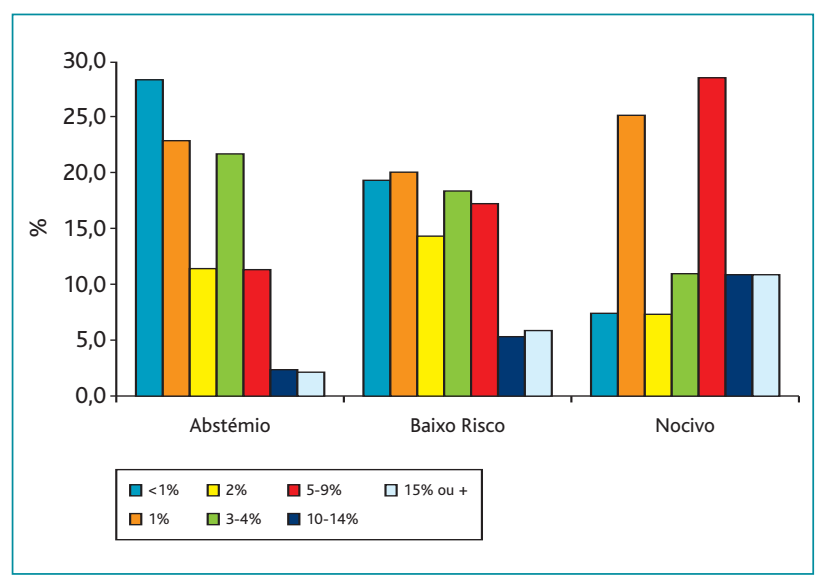

Figura 3. Distribuição dos inquiridos, considerando o tipo de consumo, pelo RCG calculado pelas tabelas SCORE

terial. As modificações induzidas pelo consumo de baixo risco de bebidas alcoólicas levariam a uma diminuição do SCORE, por diminuição do colesterol total e da pressão arterial.

No cálculo do RGC foi utilizado o colesterol total, e não o rácio colesterol total/HDL-colesterol, uma vez que, para além de a determinação do colesterol total ser mais simples e realizável, as tabelas SCORE que utilizam o rácio HDL/Colesterol total têm resultados muito semelhantes às que usam somente o colesterol total. ${ }^{6}$

Comparando os resultados obtidos com os disponíveis para a população portuguesa, ${ }^{13}$ constatou-se na

\begin{tabular}{|c|c|c|c|c|}
\hline \multicolumn{5}{|c|}{$\begin{array}{l}\text { QUADRO VI. Distribuição dos indivíduos da amostra, } \\
\text { segundo os tipos de consumo de bebidas alcoólicas, } \\
\text { por factores de risco cardiovascular modificáveis }\end{array}$} \\
\hline & Abstémio & $\begin{array}{c}\text { Baixo } \\
\text { risco }\end{array}$ & Nocivo & \\
\hline \multicolumn{5}{|l|}{ Tabaco } \\
\hline Fumador & 12,3 & 22,1 & 38,2 & $p=0,01^{*}$ \\
\hline Não Fumador & 87,7 & 77,9 & 61,8 & \\
\hline \multicolumn{5}{|l|}{ PA sistólica } \\
\hline$\leq 140 \mathrm{mmHg}$ & 63,6 & 51,3 & 26,5 & $p=0,01^{*}$ \\
\hline$>140 \mathrm{mmHg}$ & 36,4 & 48,7 & 73,5 & \\
\hline \multicolumn{5}{|l|}{ Colesterol Tot. } \\
\hline$\leq 200 \mathrm{mg} / \mathrm{dL}$ & 65,7 & 68,4 & 58,6 & $p=0,581^{*}$ \\
\hline$>200$ mg/dL & 34,3 & 31,6 & 41,4 & \\
\hline
\end{tabular}

*teste do $\chi^{2}$ 
população estudada uma menor percentagem de indivíduos com história de consumo de bebidas alcoólicas, alguma vez na vida $(51,8 \%$ versus $76 \%)$. Relativamente aos consumidores habituais, aqueles que consumiram no último ano, a diferença não é tão grande: $46 \%$ na população dos utilizadores do CSBC versus $59 \%$ na população portuguesa. Estas diferenças podem ser devidas ao diferente número de homens nas duas populações, sendo que em ambas é entre estes que se verificam as percentagens mais elevadas de consumo.

Assim como na população portuguesa, na população estudada a idade do primeiro contacto situa-se maioritariamente entre os 15 e os 19 anos de idade, principalmente pela grande percentagem de homens que iniciam o consumo nestas idades. Verifica-se também que, tal como na população portuguesa, existe um segundo pico entre os 20 e os 24 anos, desta vez com maior número de mulheres.

Quanto à duração do consumo, a população do estudo apresenta uma duração média de consumo superior à da população portuguesa, o que se pode dever ao facto de a população estudada ser composta em maior proporção pelos grupos etários com mais de 35 anos.

Na população de utilizadores do CSBC, o consumo de baixo risco de bebidas alcoólicas não se mostrou associado a um RGC inferior ao dos abstémios. No entanto, este risco foi inferior ao dos consumidores de risco e nocivo de bebidas alcoólicas. Estes resultados apoiam a hipótese de a curva dose-resposta para o consumo de bebidas alcoólicas ser em J e não em U, ou seja, não existiria um ponto em que o consumo de baixo risco ofereça benefícios relativamente à ausência de consumo de bebidas alcoólicas ou consumos inferiores, tal como referido em outros estudos. ${ }^{14}$

A associação entre o consumo de bebidas alcoólicas e o RGC calculado com o SCORE, pode ser devida à associação observada entre o alcoolismo e idade do utente e/ou o sexo masculino. Ambos contribuem para o cálculo do SCORE, sendo o seu peso, neste cálculo, superior aos possíveis efeitos benéficos que seriam observáveis com o consumo de baixo risco pela diminuição do colesterol e da pressão arterial.

Relativamente aos factores de risco modificáveis utilizados para o cálculo do SCORE (pressão arterial, hábitos tabágicos e colesterol total), verifica-se que a proporção de indivíduos com pressão arterial aumentada e hábitos tabágicos actuais aumenta com o aumento do consumo de bebidas alcoólicas, sendo esta associação estatisticamente significativa. Não se verifica uma diminuição da pressão arterial entre os consumidores de baixo risco, como seria de esperar caso se verificasse um efeito protector cardiovascular com o consumo de baixo risco de bebidas alcoólicas.

Entre os factores de risco modificáveis, apenas a colesterolemia capilar não se encontra associada ao aumento do consumo de bebidas alcoólicas. Neste parâmetro é observável uma menor proporção de indivíduos com colesterolemia superior a $200 \mathrm{mg} / \mathrm{dL}$, entre os consumidores de baixo risco. Embora não tenha sido testada a sua relevância em termos estatísticos, as diferenças encontradas estão de acordo com as opiniões divulgadas sobre os benefícios do consumo de baixo risco de bebidas alcoólicas. No entanto, este parâmetro não tem muito peso no cálculo do RGC pelas tabelas SCORE, pelo que o efeito cardioprotector do consumo de baixo risco de bebidas alcoólicas não se reflecte nos resultados do SCORE.

Os valores obtidos neste estudo para a população de utentes utilizadores do CSBC são, em muitos parâmetros, diferentes dos valores da população nacional, o que é observável logo a partir da descrição da amostra, estando os homens e os grupos etários mais jovens subrepresentados. Assim, os resultados não são extrapoláveis para a população portuguesa; no entanto, poderão ser válidos para as populações de utentes utilizadores de outros Centros de Saúde.

Durante a colheita de dados, deparamo-nos com utentes que referiram ter iniciado o consumo de bebidas alcoólicas após os 60 anos, por conselho médico, devido aos seus efeitos cardioprotectores.

Face aos resultados deste estudo, parece mais prudente não aconselhar o início do consumo de bebidas alcoólicas pelos seus benefícios a nível cardiovascular, e recomendar a diminuição deste consumo aos consumidores habituais.

\section{REFERÊNCIAS BIBLIOGRÁFICAS}

1. Mello ML, Barrias JC, Breda JJ. Álcool e problemas ligados ao álcool em Portugal. Lisboa: Direcção-Geral da Saúde; 2001.

2. Stampfer MJ, Colditz GA, Willett WC, Speizer FE, Hennekens CH. A prospective study of moderate alcohol consumption and the risk of coronary disease and stroke in women. N Engl J Med 1988 Aug 4; 319 (5): 267-73. 
3. Sesso HD, Stampfer MJ, Rosner B, Hennekens CH, Manson JE, Gaziano JM. Seven-year changes in alcohol consumption and subsequent risk of cardiovascular disease in men. Arch Intern Med 2000 Sep 25; 160 (17): 2605-12.

4. de Labry LO, Glynn RJ, Levenson MR, Hermos JA, LoCastro JS, Vokonas PS. Alcohol consumption and mortality in an American male population: recovering the U-shaped curve - findings from the normative aging study. J Stud Alcohol 1992 Jan; 53 (1): 25-32.

5. Providência R. Protecção cardiovascular por bebidas alcoólicas: bases científicas do paradoxo francês. Rev Port Cardiol 2006 Nov; 25 (11): 1043-58.

6. Conroy R, Pyörälä K, Fitzgerald AP, Sans S, Menotti A, De Baker G, et al. Estimation of ten-year risk of fatal cardiovascular disease in Europe: the SCORE project. Eur Heart J 2003 Jun; 24 (11): 987-1003.

7. McWinney I. Manual de medicina familiar. Lisboa: Inforsalus; 1994.

8. Ontario Heart Health Survey. Disponível em: http://prod.library.utoronto.ca:8090/datalib/codebooks/utm/canheart/SURVEY/ont.pdf [acedido em 05/03/2009].

9. Risco Global Cardiovascular. Circular Normativa da DGS $N^{\circ}: 06 / D S P C S$. Lisboa: Direcção-Geral da Saúde; 2007.

10. Graham I, Atar D, Borch-Johnsen K, Boysen G, Burell G, Cifkova R, et al. European guidelines on cardiovascular disease prevention in clinical practice. Fourth Joint Task Force of the European Society of Cardiology and the other Societies on cardiovascular disease prevention in clinical practice. Eur J Cardiovasc Prev Rehabil 2007 Sep, 14 Suppl 2: S1-S113.

11. Reis E, Melo P, Andrade R, Calapez T. Estatística Aplicada. Lisboa: Edições Sílabo; 1996.
12. Pestana M, Gageiro J. Análise de dados para as ciências sociais, a complementaridade do SPSS. 4 a edição. Lisboa: Edições Sílabo; 2005.

13. Inquérito Nacional ao Consumo de Substâncias Psicoactivas na População Portuguesa. Lisboa: CEOS - Investigações Sociológicas Universidade Nova de Lisboa; 2001.

14. White IR, Altmann DR, Nanchahal K. Alcohol consumption and mortality: modelling risks for men and women at different ages. BMJ 2002 Jul 27; 235 (7357): 191.

Conflitos de Interesse: não assinalados

\section{AGRADECIMENTOS}

Ao Departamento de Saúde Pública do CSBC, pelo fornecimento de toda a informação requisitada.

À Junta de Freguesia de Canidelo, pela impressão dos questionários. Aos Laboratórios Pfizer Lda., pelo fornecimento dos meios de medição do colesterol capilar.

\section{ENDEREÇO PARA CORRESPONDÊNCIA}

Martinho José Frois Almeida

Telemóvel 965581360

R. Capitão Galhardo 59 A 3 DF, Gulpilhares, 4405-000 Gulpilhares VNG E-mail:martifrois@gmail.com

Recebido em 15/01/2009

Aceite para publicação em 30/03/2009

\begin{abstract}
Objectives: To characterize drinking patterns of users of the Health Centre Barão do Corvo (CSBC) and verify whether there is an association between moderate alcohol habits and overall cardiovascular risk (RGC), calculated by the SCORE charts.

Type of study: Observational study, cross-sectional analysis.

Location: General and Family Medicine ambulatory care centre.

The study population: Users of the CSBC aged $\geq 18$ years.

Methods: Collection of data through personal interviews in May and June 2007, in the CSBC, Vila Nova de Gaia. Level of significance: 0.05 . Convenience sample $(n=502)$. The ?2 test was utilized.

Results: $51.8 \%$ of the respondents consumed alcohol once in life, and $46 \%$ in the previous year. Consumption is higher among the male gender, older people, pensioners, widows and in the less educated. $63.7 \%$ of the respondents started the consumption before the age of $20.39 .6 \%$ were moderate consumers, and $6.8 \%$ heavy consumers. The proportion of respondents with RGC above $5 \%$, increased with the consumption of alcohol.

Conclusion: Compared with the population, there is less consumption of alcohol once in life $(51.8 \%$ vs $76 \%)$ and in the previous year ( $46 \%$ vs $59 \%$ ). As in our population, age of first contact is located mostly between the age of 15 and 19 years. The average consumption is higher than the general population. The results support the hypothesis of dose-response curve for the consumption of alcohol be in $\mathrm{J}$ and not in $\mathrm{U}$ shape. It seems more prudent not to advise the consumption of alcohol for its cardiovascular benefits and to recommend the reduction of consumption for usual consumers.
\end{abstract}

Keywords: Alcohol Habits; Cardiovascular Protection. 